Article

\title{
Shaping Planetary Nebulae with Jets and the Grazing Envelope Evolution
}

\author{
Noam Soker ${ }^{1,2}$ iD \\ 1 Department of Physics, Technion, Haifa 3200003, Israel; soker@physics.technion.ac.il \\ 2 Guangdong Technion Israel Institute of Technology, Shantou 515069, China
}

Received: 14 February 2020; Accepted: 13 March 2020; Published: 18 March 2020

check for updates

\begin{abstract}
I argue that the high percentage of planetary nebulae (PNe) that are shaped by jets show that main sequence stars in binary systems can accrete mass at a high rate from an accretion disk and launch jets. Not only does this allow jets to shape PNe, but this also points to the importance of jets in other types of binary systems and in other processes. These processes include the grazing envelope evolution (GEE), the common envelope evolution (CEE), and the efficient conversion of kinetic energy to radiation in outflows. Additionally, the jets point to the possibility that many systems launch jets as they enter the CEE, possibly through a GEE phase. The other binary systems in which jets might play significant roles include intermediate-luminosity optical transients (ILOTs), supernova impostors (including pre-explosion outbursts), post-CEE binary systems, post-GEE binary systems, and progenitors of neutron star binary systems and black hole binary systems. One of the immediate consequences is that the outflow of these systems is highly-non-spherical, including bipolar lobes, jets, and rings.
\end{abstract}

Keywords: planetary nebulae: general; ISM: jets and outflows; stars: AGB and post-AGB; binaries: general; stars: mass-loss; stars: winds; stars: outflows

\section{Introduction}

The early recognition that jets play major roles in shaping planetary nebulae (PNe; e.g., [1-3]) has received great attention and support in recent years (e.g., [4-11]). The binary system might launch jets before and/or after the common envelope evolution (CEE; e.g., [12]). In any case, in most cases the jets are coeval with the main nebula [13]. The precessing of jets in many cases, e.g., as in Fleming 1 [14], show that the launching mechanism is a precessing accretion disk around a compact star, such as a main sequence star or a white dwarf (WD); or the core of the asymptotic giant branch (AGB) progenitor of the PN, rather than a collimation by a large-scale equatorial torus or by the AGB envelope.

To form an accretion disk that might efficiently launch jets, the accretion flow should have a large, specific angular momentum. Binary interaction, mostly mass transfer, must supply the angular momentum in evolved stars. Indeed, observations and their interpretations indicate that most $\mathrm{PNe}$ with axisymmetrical and/or point-symmetrical morphological features are born in binary systems (e.g., [15-23]).

There are some general properties in the operation of jets which I take to hold in shaping PNe ([24] for a review of all these properties). (1) The jets' velocity is about the escape speed of the object that launches the jets. For main sequence companions, the most common companion in shaping $\mathrm{PNe}$, the escape speed, is $v_{\mathrm{esc}} \simeq 600 \mathrm{~km} \mathrm{~s}^{-1}$, implying jet velocities in the general range of $v_{\mathrm{j}} \simeq(0.5-2) v_{\mathrm{esc}} \simeq 300-1200 \mathrm{~km} \mathrm{~s}^{-1}$. (2) The jets influence the gas in the ambient medium by depositing energy and momentum to the gas; i.e., by heating and/or expelling it. As such, there is less mass available for accretion onto the object that launches the jets, therefore reducing the jets' power. Namely, the jets operate in a negative feedback cycle. In particular, the jets might shut themselves down by removing the gas available for accretion, 
and resume activity later on as accretion renews. This might repeat itself several times (the different mass ejection episodes will merge later to one nebula). (3) Alongside the negative feedback cycle, there is a positive feedback cycle due to the jets. As jets are likely to remove energy and high entropy gas, they allow more gas to flow-in, and that way increase the mass accretion rate (e.g., [25]). Chamandy et al. [26] removed mass and pressure via a subgrid mechanism, and showed that this "pressure release valve" mechanism, as jets are expected to act, allows high accretion rates. (4) The jets are not necessarily narrow. Jets might be wide, with a half-opening angle of almost $90^{\circ}$ (e.g., observations by [27]).

Several studies have used post-CEE binary central stars of PNe to constrain the physics and parameters of the CEE (e.g., [28,29]). Jones (2020; [29]) presents a thorough review on the usage of post-CEE PNe to learn about the CEE. Here, I concentrate on the role of jets and use some properties of PNe and post-AGB binary systems to learn about the importance and outcome of the grazing envelope evolution (GEE).

\section{The Grazing Envelope Evolution (GEE)}

In the GEE (suggested in 2015 [30]), the jets that the secondary star launches manage to eject the envelope outside its orbit (for numerical simulations see, e.g., [31-33]). During the GEE, the secondary star grazes the envelope, including being somewhat outside the photosphere or somewhat inside it. The accretion process of gas from the giant envelope to the companion is a combination of Bondi-Hoyle-Lyttleton (BHL) accretion flow from the surrounding gas, and a Roche lobe overflow (RLOF) from the inner dense envelope. Rather than directly entering the CEE, the system performs the GEE. It might later enter a CEE, or not. In some cases the secondary might get into the envelope and then exit back (e.g., [34]). This process might repeat itself.

The GEE phase has several effects and outcomes which I list as follows.

\subsection{Preventing the CEE}

By removing mass at a high rate, the jets might prevent the CEE altogether (e.g., [35]). This process works in a negative feedback cycle, as when the orbital separation increases, the accretion rate decreases, and so does the effect of the jets. Tidal forces then bring the system back into contact, resuming accretion at a high rate and so on. The outcome might be a binary system with an intermediate orbital separation, $a \approx 1 \mathrm{AU}$. There is a group of post-AGB binary systems with such orbital separations; in some, the companion is observed to launch jets (e.g., $[27,36,37])$. The GEE, therefore, might account for these post-AGB intermediate binaries (post-AGBIBs; [35]).

\subsection{Postponing the CEE and Removing Mass before the CEE}

In some cases the GEE does not prevent the CEE, but postpones it and as a result of that the system loses large amount of mass before entering the CEE. Even without launching jets, many binary systems can lose large amounts of mass before entering the CEE (e.g., [38,39]). Jones [29] concludes in his review that both post-CEE PNe and merging stars (luminous red novae) strongly suggest that appreciable mass transfer/loss occurs before the onset of the CEE.

\subsection{Shaping the Outflow}

The interaction of the jets with the outskirts of the envelope of the giant star leads to a complicated flow structure (e.g., [32,40]). In this phase, the jets mostly interact with the envelope outskirts and with the dense base of the wind, and do not expand to large distances (Section 3).

\subsection{Counteracting Tidal Circularization}

Substantially enhanced mass-loss at periastron passages during the GEE can counteract the circularization effect due to tidal interactions [41]. This might account for the non-zero eccentricity of binary systems, such as post-AGBIBs. Additionally, it might lead some systems to enter a CEE while they have high eccentricity. 
One outcome of such a process might be a nebula that possesses departure from pure axisymmetrical structure (e.g., [42] and examples therein).

\subsection{Experiencing ILOT Events}

The jets that the companion launches collide with envelope gas, and if the jets penetrate out they might collide with the shell that the high mass loss rate from the giant star forms. The collision of the jets with the envelope (e.g., [43]) and/or with the slow shell (e.g., [44]) converts kinetic energy to thermal energy. If the photon diffusion time out is not much longer than the expansion time of the gas (which is the adiabatic cooling time), then a large fraction of the thermal energy is carried out by radiation. In a short GEE episode, less than few years, the observation will be of an intermediate-luminousity optical transient—an ILOT (which might be brightest in the IR if efficient dust formation takes place).

\subsection{Forming Type IIb Supernovae (SNe IIb)}

$\mathrm{SNe} \mathrm{Ilb}$ are core-collapsed supernovae with little hydrogen mass at explosion $\left(M_{\mathrm{H}, \mathrm{ex}} \simeq 0.01-1 M_{\odot}\right)$. The enhanced mass loss due to jets in a binary system of a giant progenitor of core-collapsed supernova and a main sequence companion that experiences the GEE, might leave little hydrogen mass at explosion; hence, forming a SN IIb progenitor [34]. This is only one of several evolutionary channels that might lead to $\mathrm{SNe} \mathrm{IIb}$.

\section{Evolutionary Phases of the Jets' Launch}

For the purpose of this study I consider five evolutionary phases where the companion to a giant star might launch jets. I summarize these in two tables. In Table $1 \mathrm{I}$ list three phases where in most cases the jets do not expand to large distances. In Table 2 I list the two phases where in most cases the jets might expand to large distances and form bipolar lobes and/or other morphological features along and near the symmetry axis.

1. Jets in wind acceleration zone (first column of Table 1). The secondary star can remove mass before it grazes the envelope itself, if it launches jets in the acceleration zone of the dense wind of the giant star, as numerical simulations show [45]. When the accretion rate is mostly due to BHL accretion flow, the jets from main sequence stars are not strong enough to penetrate through the wind from which the secondary star accretes mass [45].

2. Jets from pre-CEE RLOF accretion (first column of Table 2). When the secondary is closer to the surface, the accretion is mainly due to RLOF from the envelope. Most of the accretion flow is equatorial and it is much larger than that of BHL accretion from the wind through which the secondary orbits the giant star. In such a case the jets are likely to penetrate through the wind and expand to large distances, thereby shaping the slow outflow to form a bipolar morphology with one or more pairs of lobes and/or other point symmetric morphologies (e.g., [46]). This case might occur also at a somewhat earlier phase when the dense acceleration zone of the wind overflows its Roche lobe and transfers mass at a high rate to the secondary star via an equatorial flow (for this wind-RLOF see, e.g., $[47,48])$.

3. Jets during the GEE (second column of Table 1). This phase was the subject of Section 2. The jets strongly interact with the envelope, and in most cases do not expand to large distances. The situation might be different in an eccentric orbit (as might have been the case during the Great Eruption of Eta Carinae; e.g., [49]), when the secondary star accretes mass when it grazes the giant envelope near periastron, and the jets' launching episode lasts at later times at which the secondary star is near apastron.

4. Jets during the CEE (third column of Table 1). Inside the envelope the density is too large for jets that a main sequence star launches to expand out (e.g., [50]). The jets might play a crucial role in helping mass removal.

5. Jets during the post-CEE (second column of Table 2). In a recent paper [51] I discussed the possibility that the secondary star accretes mass from a circumbinary disk [52-54] at the termination 
of the CEE. As a result of that, the secondary star might launch jets that have variable directions and intensities. These jets shape the nebula. Note that in this mechanism the secondary star (or maybe even the core of the former AGB star) launches the jets. This should be distinguished from a process wherein the highly deformed envelope collimates a bipolar outflow (e.g., $[55,56])$.

Table 1. Evolutionary phases with choked jets.

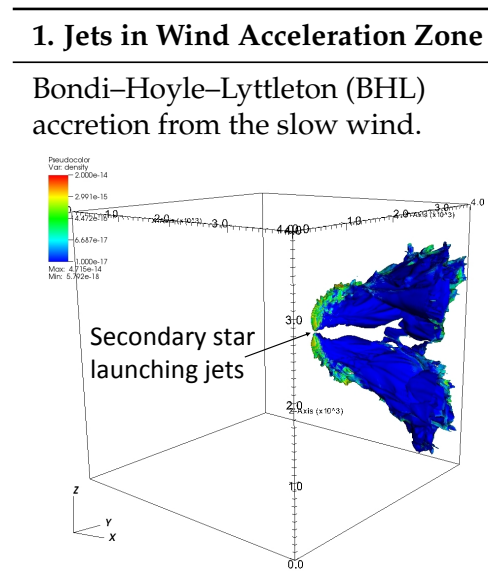

Density-surface 3D maps of the gas that originated in the jets and mixed with the wind. Colours are density surfaces according to the colour bars on the left from $10^{-17}$ (blue) to $2 \times 10^{-14} \mathrm{~g} \mathrm{~cm}^{-3}$ (red) (from [45]).

\section{Jets during the GEE}

BHL + Roche lobe overflow (RLOF) accretion from envelope outskirts.

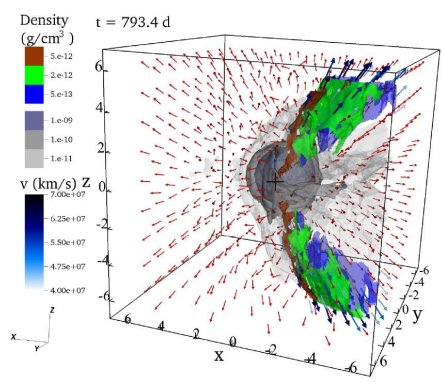

The asymptotic giant branch (AGB) is at the center. The secondary star (marked by " + ") is moving to the left in the figure. The red-green-blue colors show gas originated in the jets and moving with velocities $>400 \mathrm{~km} \mathrm{~s}^{-1}$.

The gray denotes slow AGB gas. Axes from $-7 \times 10^{13}$ to $7 \times 10^{13} \mathrm{~cm}$. (For more details, see the original study [40]).

\section{Jets during the CEE}

BHL accretion inside the giant envelope.

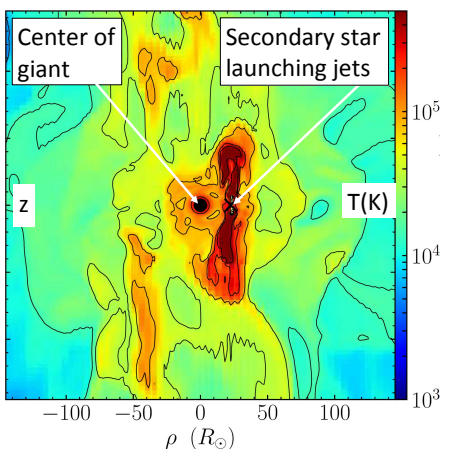

A temperature map (bar in $\mathrm{K}$ ) in the meridional plane of a 3D CEE simulation. The secondary launches two opposite jets, in the $+z$ and $-z$ directions, as it orbits inside the envelope of a giant star. The high temperature (red) of the shocked jets' gas shows the jets do not break out from the envelope; i.e., they are choked jets (from [50]).

Table 2. Evolutionary phases with expanding jets.

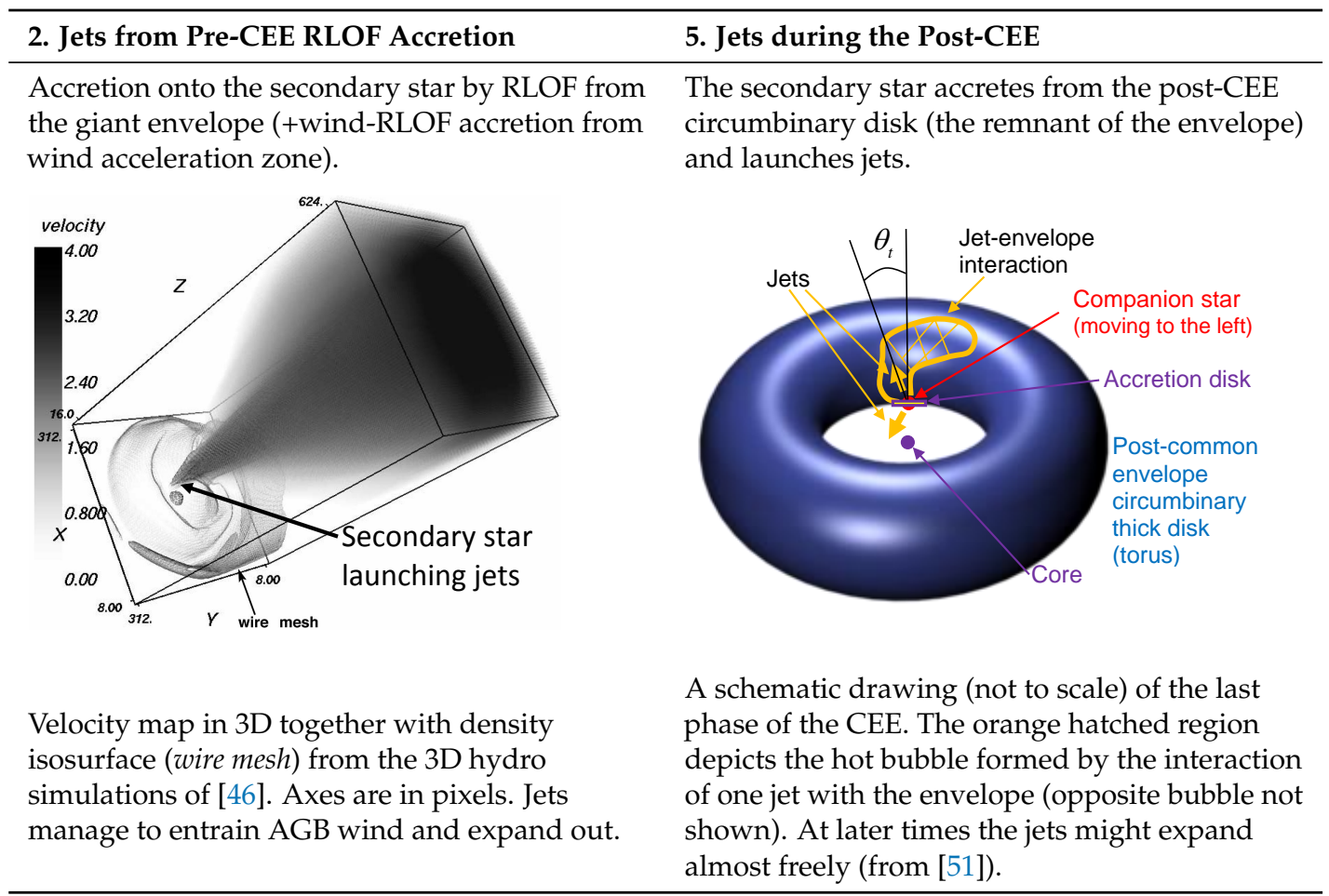




\section{Supporting observations to the GEE}

In this section I list some observations that support to some extent the occurrence of the GEE. There is no observation (yet) that directly shows this phase takes place, but I take the observations I list in Table 3 to indirectly support the GEE. These observations are of two kinds: those that show the importance of jets (first four items of Table 3), and those that raise some puzzles that the GEE might solve (last two rows of Table 3).

Table 3. Observational indirect support for the importance of the GEE.

\begin{tabular}{|c|c|c|}
\hline Property & Example & Possible Implications \\
\hline $\begin{array}{l}\text { Jets launching } \approx 10^{3}-10^{4} \mathrm{yr} \\
\text { before main nebular ejection. }\end{array}$ & ETHOS $1[20]$ & $\begin{array}{l}\text { A strong binary interaction which involves mass } \\
\text { transfer and jet launching takes place shortly before } \\
\text { the CEE. Might be phase } 2 \text { (left column) of Table } 2 \text {. }\end{array}$ \\
\hline $\begin{array}{l}\text { Jets launching } \approx 10^{3}-10^{4} \mathrm{yr} \\
\text { after main nebular ejection. }\end{array}$ & $\mathrm{Hb} 4[7]$ & $\begin{array}{l}\text { A strong binary interaction which involves mass } \\
\text { transfer and jet launching takes place shortly after the } \\
\text { CEE. Might be mass accretion from a circumbinary } \\
\text { disk, as phase } 5 \text { in Table } 2 \text {. }\end{array}$ \\
\hline $\begin{array}{l}\text { High-momentum jets in } \\
\text { pre-PNe (or in PNe). }\end{array}$ & M1-92 [57] & $\begin{array}{l}\text { Main sequence stars can accrete mass at high rates, } \\
\dot{M}>10^{-4} M_{\odot} \mathrm{yr}^{-1} \text {, and launch jets when } \\
\text { approaching, inside, or just exiting a CEE [58]. }\end{array}$ \\
\hline $\begin{array}{l}\text { Post-AGBIBs with jets and a } \\
\text { circumbinary disk. }\end{array}$ & $\begin{array}{l}\text { IRAS 19135+3937 } \\
\text { [27] }\end{array}$ & $\begin{array}{l}\text { (1) Main sequence companion outside an AGB star } \\
\text { can launch jets that shape the outflow. (2) Accretion } \\
\text { might take place from a circumbinary disk [27]. }\end{array}$ \\
\hline $\begin{array}{l}\text { Low-mass nebulae in some } \\
\text { post-CEE PNe [59]. }\end{array}$ & Abell 63 [60] & Significant pre-CEE mass loss; possibly in a GEE [59]. \\
\hline $\begin{array}{l}\text { PN central binaries with orbital } \\
\text { periods of } P_{\text {orb }}>\text { several day. }\end{array}$ & NGC 2346 [61] & Significant pre-CEE mass loss; possibly in a GEE [30]. \\
\hline
\end{tabular}

Jets launching $\approx 10^{3}-10^{4} \mathrm{yr}$ before or after main nebular ejection. Observations suggest that in some cases PN progenitors launch jets shortly before, during, or after they eject the main nebulae (e.g., $[12,13,62,63])$. In the second and third rows of Table 3, I list two examples and summarize the possible implications of these observations.

High momentum jets in PNe. Blackman \& Lucchini [58] studied 19 pre-PNe (taken from [57,64]) and constrained the minimum mass accretion rate to account for the high momenta of the bipolar outflows (jets). They considered main sequence and white dwarf (WD) accretors to launch the jets. They concluded that accretion from the wind is not sufficient to explain the momentum in the jets. Main sequence companions accreting via a RLOF or inside a common envelope can supply the required momentum. As for WD accretors, in most cases that they studied, the accretion rate was super-Eddington. As I discuss in Section 5, another problem with WDs accreting at a high rate is that they ignite nuclear reactions and inflate an envelope. Overall, the conclusion from the work of [58] is that main sequence stars can accrete at high rates, $\dot{M}>10^{-4} M_{\odot} \mathrm{yr}^{-1}$, just before, during, and/or just after the CEE (third item in Table 3).

Post-AGBIBs with jets and a circumbinary disk. Traditional CEE calculations suggest that either the binary system enters a CEE and the final orbital separation is much smaller than the giant radius $(\approx 1 \mathrm{AU})$, or that mass loss causes the orbital separation to increase much above the giant radius. However, there is a group of post-AGB intermediate binaries (post-AGBIBs) with orbital separations of $\approx 1$ AU. I already mentioned this group in Section 2.1, in which I suggested that the GEE might prevent the formation of a CEE, such that the orbital separation stays at about the giant radius. The point I emphasize here is that observations suggest that the main sequence companion in most post-AGBIBs launches jets (e.g., [27,36,37]). I list the implications in the fourth item of Table 3 . The second implication, 
that the companion accretes mass from a circumbinary disk and launches jets, is directly relevant to post-CEE binaries, as indicated in the second column of Table 2.

Low-mass nebulae in some post-CEE PNe. There are several hints that a large fraction of systems enter the CEE after they have lost a significant amount of mass (e.g., [29] for a recent paper). Santander-García [59] as another example, claim lower nebular mass in post-CEE PNe. This again suggests a substantial pre-CEE mass loss, which the GEE might account for [59]. Many episodes of GEE might help with removing large amounts of mass. Another outcome of significant pre-CEE mass loss might be a large post-CEE orbital separation [30], such as in the central binary system of NGC 2346.

$P N$ central binaries with orbital periods of $P_{\text {orb }}>$ several days. Central binary systems of PNe with orbital separations below $\approx 100 R_{\odot}$ have experienced the CEE (rather than only the GEE for larger orbital separations). If the orbital separation is above $\approx 10 R_{\odot}$, corresponding to an orbital period of $P_{\text {orb }}>4$ days, the possible implication is that the envelope mass at the beginning of the CEE was small, such that the binary system ejected the entire envelope when the orbital separation was relatively large. The GEE might be a way to remove mass in the pre-CEE phase.

\section{The Mass-Accreting Secondary Star}

In earlier sections I referred mainly (but not only) to main sequence secondary stars. Here I list also other possibilities. I list by increasing maximum jet power.

\subsection{Planets as Secondary Objects}

In cases of low mass PN progenitors where the final envelope mass is low, $M_{\text {env }} \simeq 0.1-0.5 M_{\odot}$, a planet can play a role in shaping the outflow [65]. This scenario is made more likely if the mass loss rate of AGB stars that do not acquire any angular momentum from a companion (so called Jsolated stars) is lower than what traditional formulae give (e.g., [66]).

Planets might accrete small amounts of mass, and at best launch jets at velocities of $\approx 100 \mathrm{~km} \mathrm{~s}^{-1}$, which is about the escape velocity of massive planets. As planets have no strong convection that mixes accreted mass inward, I take the mass that a planet of mass $M_{\mathrm{p}}$ can accrete before it expands to be below about $M_{\mathrm{acc}, \mathrm{p}} \simeq 0.01-0.1 M_{\mathrm{p}}$; this number is a crude estimate (a guess even), and it requires further detailed studies. The mass in each jet might be about 10 percent of the accreted mass (as in young stellar objects; e.g., [67]), $M_{\text {jet,p }} \approx 0.001-0.01 M_{\mathrm{p}}$. Scaling this relation with a massive planet (about 10 times the mass of Jupiter) gives $M_{\text {jet }} \approx 10^{-5}-10^{-4}\left(M_{\mathrm{p}} / 0.01 M_{\odot}\right) M_{\odot}$. It is possible that if the planet accretes more mass it expands such that it does not launch jets anymore.

Interestingly, there are two opposite knots along the symmetry axis of some elliptical PNe, termed "ansae" (or FLIERS), whose typical velocities and masses are $\approx$ few $\times 10-200 \mathrm{~km} \mathrm{~s}^{-1}$ and $\approx 10^{-5}-10^{-4} M_{\odot}$, respectively [68]. There is a need for more studies to examine whether massive planets and brown dwarfs might launch jets in shaping PNe, and whether in some cases these jets might form ansae (FLIERS).

There is an indirect way for a planet (or a brown dwarf) to form jets. If the core of the AGB star tidally destroys the planet to form an accretion disk at the termination of the CEE, then the core might launch jets made from the destroyed planet material (e.g., [69-73]). These jets, though, will be much faster than the observed velocities of ansae.

\subsection{WD Secondary Stars}

In [74] I discuss accreting WDs and main sequence stars during the CEE (note that here the WD is the secondary star, and the mass-donor giant is the primary star). The potential well of a WD is about two orders of magnitude deeper than that of a main sequence star. However, the mass accretion rate of a WD is limited. This is because at an accretion rate of $\dot{M}_{\mathrm{WD}}>\approx 10^{-6} \mathrm{M}_{\odot} \mathrm{yr}^{-1}$ nuclear reactions on the surface of the WD inflate an envelope (e.g., [75]). This upper limit is about the lower accretion rate that [58] is required to explain high-momenta bipolar outflows in pre-PNe. 
This addition of nuclear energy is about a doublin ofg the luminosity from the core of the AGB star. It is not clear whether the WD can launch jets at accretion rates above that value. Still, at lower values of $\dot{M}_{\mathrm{WD}}<$ few $\times 10^{-7} M_{\odot} \mathrm{yr}^{-1}$, the WD might launch jets at terminal velocities of $v_{\mathrm{j}, W \mathrm{D}} \approx \mathrm{few} \times 1000 \mathrm{~km} \mathrm{~s}^{-1}$. Overall, WDs might launch jets at the different stages that I summarized in Section 3 and play a role in the different phases. I expect jets from WDs not to reach the highest powers of jets from main sequence stars. This question deserves further study.

\subsection{Main Sequence Secondary Stars (Low Masses and High Masses)}

The supporting observations that I discussed in Section 4 are mainly for main sequence secondary stars in low mass systems. Namely, the secondary stars are mainly in the mass range $\simeq 0.3-1 M_{\odot}$, and observations suggest that these secondary stars can accrete at a high rate and launch jets. These are main sequence stars with convective envelopes. They are more likely to accrete mass without expanding much. The removal of angular momentum, energy, and high entropy gas by the jets themselves [25], as in the "pressure release valve" mechanism [26], helps in allowing high mass accretion. There is no question that low-mass main sequence stars can launch relatively energetic jets.

I emphasize that observations (e.g., [76]) do show that post-CEE main sequence stars have larger radii than their radii on the main sequence, as expected for such stars that accrete mass (e.g., [77]). The point is that the radii of these post-CEE main sequence stars increase by a factor of only $\approx 2$. Namely, the gravitational potential of the secondary star does not change much, and the escape velocity is still larger than $\approx 300 \mathrm{~km} \mathrm{~s}^{-1}$. Namely, jets that these stars launch even after they expand are still relatively fast, and they might be sufficiently energetic to shape the nebula and expel mass from the envelope.

There is the question of whether more massive stars that have radiative envelopes can accrete mass and launch jets. I think that the bipolar nebula of Eta Carinae, called Homunculus, which was formed during the Great Eruption in the nineteenth century, is evidence that the answer is yes. There are indications and suggestions (e.g., [78] and references therein) that the Homunculus was shaped by jets. As [78] showed, jets that the secondary star launched might as well account for the very fast outflow in the Great Eruption $\left(v>10^{4} \mathrm{~km} \mathrm{~s}^{-1}\right)$.

\subsection{Neutron Stars and Black Holes}

In principle, a neutron star (NS) and a black hole (BH) can be the secondary star in each of the five jet-launching phases that I discussed in Section 3. In particular, during the GEE and the CEE, neutrino cooling allows very high mass accretion rates. The giant primary star is likely to be a massive progenitor of a core-collapsed supernova with a mass above $\approx 8 M_{\odot}$. The role of jets that a NS and a $\mathrm{BH}$ launch during the CEE (and possibly in an earlier GEE phase) must be considered in the study of the formation of NS-NS, NS-BH, or BH-BH close binary systems.

The collision of jets that main sequence stars (and possibly WDs) launch with the envelope and slow outflow can lead to ILOTs; i.e., transient events that have luminosities below the typical luminosity of a supernova. On the other hand, the collision of the very energetic jets that NSs and BHs launch during the CEE can convert huge amounts of energy to thermal energy, part of which is radiated away, possibly as an event as bright as a typical supernova or more. If the NS (or $\mathrm{BH}$ ) spirals-in all the way to the core, it destroys the core and might accrete a mass of $M_{\text {acc,Ns }} \approx 0.1-1 M_{\odot}$ within minutes [79]. The outcome might be a very bright event, much brighter even than typical supernovae (e.g., [80]). This event is termed a common envelope jets supernova (CEJSN). One plausible outcome might be the nucleosynthesis of r-process elements in the jets [79].

If the NS or BH accretes mass only from the relatively low density envelope, and then gets out without completely destructing the giant star, the event is termed CEJSN impostor [81]. 


\section{Summary}

The observations that jets that binary systems launch shape many PNe (Section 1), before, during, and/or after the ejection of the main nebula (Section 3), suggest that the companion might launch jets also-when it enters the envelope and when inside the envelope (Section 4). Therefore, when studying the CEE, it is mandatory to consider jets; in particular, when the companion is a NS or a BH (Section 5.4). These observations support the idea of the GEE (Section 4), wherein the jets manage to remove the envelope outside the orbit of the secondary star (Section 2). The secondary stars might be planets (or brown dwarfs), main sequence stars, WDs, NSs, and BHs (Section 5). This study was mainly on main sequence secondary stars.

It is important to emphasize that it is very difficult, and even impossible at this stage to fully capture the physics near the origin of the jets with numerical simulations. Numerical simulations on the scale of binary interaction cannot resolve the accretion flow and cannot self-consistently launch jets. All relevant numerical simulations of jets use a sub-grid prescription to inject jets into the numerical grid (namely, jets' injection by hand).

Finally, $\approx 10 \%$ of the systems that experience the CEE might be close triple systems. This might lead to the formation of "messy nebulae"-i.e., nebulae that lack any clear symmetry—by launching inclined jets [82].

Funding: Israel Science Foundation, grant number 420/16 and Asher Space Research Fund.

Acknowledgments: I thank Bruce Balick and the referees for very helpful comments. This research was supported by a grant from the Israel Science Foundation and a grant from the Asher Space Research Fund at the Technion.

Conflicts of Interest: The author declares no conflict of interest.

\section{References}

1. Morris, M. Mechanisms for mass loss from cool stars. Publ. Astron. Soc. Pac. 1987, 99, 1115. [CrossRef]

2. Sahai, R.; Trauger, J.T. Multipolar Bubbles and Jets in Low-Excitation Planetary Nebulae: Toward a New Understanding of the Formation and Shaping of Planetary Nebulae. Archit. J. 1998, 116, 1357. [CrossRef]

3. Soker, N. On the Formation of Ansae in Planetary Nebulae. Archit. J. 1990, 99, 1869. [CrossRef]

4. Akashi, M.; Soker, N. The formation of 'columns crowns' by jets interacting with a circumstellar dense shell. Mon. Not. R. Astron. Soc. 2018, 481, 2754-2765. [CrossRef]

5. Balick, B.; Frank, A.; Liu, B. Models of the Mass-ejection Histories of Pre-planetary Nebulae. III. The Shaping of Lobes by Post-AGB Winds. Astrophys. J. 2019, 877, 30. [CrossRef]

6. Balick, B.; Frank, A.; Liu, B. Models of the Mass-ejection Histories of Pre-planetary Nebulae. IV. Magnetized Winds and the Origins of Jets, Bullets, and FLIERs. Astrophys. J. 2020, 889, 13. [CrossRef]

7. Derlopa, S.; Akras, S.; Boumis, P.; Steffen, W. High-velocity string of knots in the outburst of the planetary nebula Hb4. Mon. Not. R. Astron. Soc. 2019, 484, 3746. [CrossRef]

8. Estrella-Trujillo, D.; Hernández-Martínez, L.; Velázquez, P.F.; Esquivel, A.; Raga, A.C. Hydrodynamical Models of Protoplanetary Nebulae Including the Photoionization of the Central Star. Astrophys. J. 2019, 876, 29. [CrossRef]

9. Rechy-García, J.S.; Guerrero, M.A.; Duarte Puertas, S.; Chu, Y. -H.; Toala, J.A.; Miranda, L.F. Kinematical investigation of possible fast collimated outflows in twelve planetary nebulae. Mon. Not. R. Astron. Soc. 2020, 492, 1957.

10. Rechy-García, J.; Velázquez, P.F.; Peña, M.; Raga, A.C. Observations and 3D hydrodynamical models of planetary nebulae with Wolf-Rayet type central stars. Mon. Not. R. Astron. Soc. 2017, 464, 2318. [CrossRef]

11. Tafoya, D.; Orosz, G.; Vlemmings, W.H.T.; Sahai, R.; Pérez-Sánchez, A.F. Spatio-kinematical model of the collimated molecular outflow in the water-fountain nebula IRAS 16342-3814. Astron. Astrophys. 2019, 629, A8. [CrossRef]

12. Tocknell, J.; De Marco, O.; Wardle, M. Constraints on common envelope magnetic fields from observations of jets in planetary nebulae. Mon. Not. R. Astron. Soc. 2014, 439, 2014. [CrossRef]

13. Guerrero, M.A.; Rechy-Garcia, J.S.; Ortiz, R. Space Velocity and Time Span of Jets in Planetary Nebulae. Astrophys. J. 2020, 890, 50. [CrossRef] 
14. Boffin, H.M.J.; Miszalski, B.; Rauch, T.; Jones, D.; Corradi, R.L.M.; Napiwotzki, R.; Day-Jones, A.C.; Köppen, J. An Interacting Binary System Powers Precessing Outflows of an Evolved Star. Science 2012, 338, 773-775. [CrossRef]

15. Bujarrabal, V.; Castro-Carrizo, A.; Van Winckel, H.; Alcolea, J.; Sánchez Contreras, C.; Santander-García, M.; Hillen, M. High-resolution observations of IRAS 08544-4431. Detection of a disk orbiting a post-AGB star and of a slow disk wind. Astron. Astrophys. 2018, 614, A58. [CrossRef]

16. Corradi, R.L.M.; Sabin, L.; Miszalski, B.; Rodríguez-Gil, P.; Santander-García, M.; Jones, D.; Drew, J.E.; Mampaso, A.; Barlow, M.J.; Rubio-Díez, M.M.; et al. The Necklace: Equatorial and polar outflows from the binary central star of the new planetary nebula IPHASX J194359.5+170901. Mon. Not. R. Astron. Soc. 2011, 410, 1349-1359. [CrossRef]

17. Jones, D. The binary central stars of planetary nebulae. In Highlights on Spanish Astrophysics X, Proceedings of the XIII Scientific Meeting of the Spanish Astronomical Society, Salamanca, Spain, 16-20 July 2018; The Spanish Astronomical Society: Salamanca, Spain, 2019; p. 340.

18. Jones, D.; Van Winckel, H.; Aller, A.; Exter, K.; De Marco, O. The long-period binary central stars of the planetary nebulae NGC 1514 and LoTr 5. Astron. Astrophys. 2017, 600, L9. [CrossRef]

19. Kővári, Z.; Strassmeier, K.G.; Oláh, K.; Kriskovics, L.; Vida, K.; Carroll, T.A.; Granzer, T.; Ilyin, I.; Jurcsik, J.; Kővári, E.; et al. Surface magnetic activity of the fast-rotating G5 giant IN Comae, central star of the faint planetary nebula LoTr 5. Astron. Astrophys. 2019, 624, A83. [CrossRef]

20. Miszalski, B.; Corradi, R.L.M.; Boffin, H.M.J.; Jones, D.; Sabin, L.; Santander-García, M.; Rodríguez-Gil, P.; Rubio-Díez, M.M. ETHOS 1: A high-latitude planetary nebula with jets forged by a post-common-envelope binary central star. Mon. Not. R. Astron. Soc. 2011, 413, 1264. [CrossRef]

21. Miszalski, B.; Manick, R.; Van Winckel, H.; Mikołajewska, J. The post-common-envelope binary nucleus of the planetary nebula IC 4776: Neither an anomalously long orbital period nor a Wolf-Rayet binary. Mon. Not. R. Astron. Soc. 2019, 487, 1040-1046. [CrossRef]

22. Orosz, G.; Gómez, J.F.; Imai, H.; Tafoya, D.; Torrelles, J.M.; Burns, R.A.; Frau, P.; Guerrero, M.A.; Miranda, L.F.; Perez-Torres, M.A.; et al. Rapidly evolving episodic outflow in IRAS 18113-2503: Clues to the ejection mechanism of the fastest water fountain. Mon. Not. R. Astron. Soc. 2019, 482, L40. [CrossRef]

23. Wesson, R.; Jones, D.; García-Rojas, J.; Boffin, H.M.J.; Corradi, R.L.M. Confirmation of the link between central star binarity and extreme abundance discrepancy factors in planetary nebulae. Mon. Not. R. Astron. Soc. 2018, 480, 4589-4613. [CrossRef]

24. Soker, N. The jet feedback mechanism (JFM) in stars, galaxies and clusters. New Astron. Rev. 2016, 75, 1-23. [CrossRef]

25. Shiber, S.; Schreier, R.; Soker, N. Binary interactions with high accretion rates onto main sequence stars. Res. Astron. Astrophys. 2016, 16, 117. [CrossRef]

26. Chamandy, L.; Frank, A.; Blackman, E.G.; Carroll-Nellenback, J.; Liu, B.; Tu, Y.; Nordhaus, J.; Chen, Z.; Peng, B. Accretion in common envelope evolution. Mon. Not. R. Astron. Soc. 2018, 480, 1898-1911. [CrossRef]

27. Bollen, D.; Kamath, D.; Van Winckel, H.; De Marco, O. A spatio-kinematic model for jets in post-AGB stars. Astron. Astrophys. 2019, 631, A53. [CrossRef]

28. De Marco, O.; Passy, J.-C.; Moe, M.; Herwig, F.; Mac Low, M.-M.; Paxton, B. On the alpha formalism for the common envelope interaction. Mon. Not. R. Astron. Soc. 2011, 411, 2277-2292. [CrossRef]

29. Jones, D. Observational Constraints on the Common Envelope Phase. arXiv 2020, arXiv:2001.03337.

30. Soker, N. Close Stellar Binary Systems by Grazing Envelope Evolution. Astrophys. J. 2015, 800, 114. [CrossRef]

31. López-Cámara, D.; De Colle, F.; Moreno Méndez, E. Self-regulating jets during the common-envelope phase. Mon. Not. R. Astron. Soc. 2019, 482, 3646. [CrossRef]

32. Shiber, S. The Morphology of the Outflow in the Grazing Envelope Evolution. Galaxies 2018, 6, 96. [CrossRef]

33. Shiber, S.; Soker, N. Simulating a binary system that experiences the grazing envelope evolution. Mon. Not. R. Astron. Soc. 2018, 477, 2584-2598. [CrossRef]

34. Naiman, B.V.; Sabach, E.; Gilkis, A.; Soker, N. Type IIb supernovae by the grazing envelope evolution. Mon. Not. R. Astron. Soc. 2020, 491, 2736. [CrossRef]

35. Abu-Backer, A.; Gilkis, A.; Soker, N. Orbital Radius during the Grazing Envelope Evolution. Astrophys. J. 2018, 861, 136. [CrossRef] 
36. Gorlova, N.; Van Winckel, H.; Ikonnikova, N.P.; Burlak, M.A.; Komissarova, G.V.; Jorissen, A.; Gielen, C.; Debosscher, J.; Degroote, P. IRAS 19135+3937: An SRd variable as interacting binary surrounded by a circumbinary disc. Mon. Not. R. Astron. Soc. 2015, 451, 2462. [CrossRef]

37. Witt, A.N.; Vijh, U.P.; Hobbs, L.M.; Aufdenberg, J.P.; Thorburn, J.A.; York, D.G. The Red Rectangle: Its Shaping Mechanism and Its Source of Ultraviolet Photons. Astrophys. J. 2009, 693, 1946. [CrossRef]

38. Bear, E.; Soker, N. Spinning-up the envelope before entering a common envelope phase. New Astron. 2010, 15, 483-490. [CrossRef]

39. MacLeod, M.; Loeb, A. Pre-Common-Envelope Mass Loss from Coalescing Binary Systems. arXiv 2020, arXiv:2003.01123.

40. Shiber, S.; Kashi, A.; Soker, N. Simulating the onset of grazing envelope evolution of binary stars. Mon. Not. R. Astron. Soc. 2017, 465, L54. [CrossRef]

41. Kashi, A.; Soker, N. Counteracting tidal circularization with the grazing envelope evolution. Mon. Not. R. Astron. Soc. 2018, 480, 3195. [CrossRef]

42. Soker, N.; Rappaport, S. Departure from Axisymmetry in Planetary Nebulae. Astrophys. J. 2001, $557,256$. [CrossRef]

43. Soker, N. Intermediate luminosity optical transients during the grazing envelope evolution (GEE). New Astron. 2016, 47, 16. [CrossRef]

44. Soker, N. Efficiently jet-powered radiation in intermediate-luminosity optical transients (ILOTS). arXiv 2020, arXiv:2001.07879

45. Hillel, S.; Schreier, R.; Soker, N. A companion star launching jets in the wind acceleration zone of a giant star. Astrophys. J. 2020, 891, 33. [CrossRef]

46. García-Arredondo, F.; Frank, A. Collimated Outflow Formation via Binary Stars: Three-Dimensional Simulations of Asymptotic Giant Branch Wind and Disk Wind Interactions. Astrophys. J. 2004, 600, 992. [CrossRef]

47. Harpaz, A.; Rappaport, S.; Soker, N. The Rings around the Egg Nebula. Astrophys. J. 1997, 487, 809. [CrossRef]

48. Mohamed, S.; Podsiadlowski, P. Wind Roche-Lobe Overflow: A New Mass-Transfer Mode for Wide Binaries. In Proceedings of the 15th European Workshop on White Dwarfs, Leicester, UK, 7-11 August 2006; Astronomical Society of the Pacific: San Francisco, CA, USA, 2007; Volume 372, p. 397.

49. Kashi, A.; Soker, N. Periastron Passage Triggering of the 19th Century Eruptions of Eta Carinae. Astrophys. J. 2010, 723, 602. [CrossRef]

50. Shiber, S.; Iaconi, R.; De Marco, O.; Soker, N. Companion-launched jets and their effect on the dynamics of common envelope interaction simulations. Mon. Not. R. Astron. Soc. 2019, 488, 5615. [CrossRef]

51. Soker, N. Variable jets at the termination of the common envelope evolution. Mon. Not. R. Astron. Soc. 2019, 483, 5020-5025. [CrossRef]

52. Chen, W.-C.; Podsiadlowski, P. Rapid Orbital Decay in Detached Binaries: Evidence for Circumbinary Disks. Astrophys. J. Lett. 2017, 837, L19. [CrossRef]

53. Kashi, A.; Soker, N. A circumbinary disc in the final stages of common envelope and the core-degenerate scenario for Type Ia supernovae. Mon. Not. R. Astron. Soc. 2011, 417, 1466-1479 . [CrossRef]

54. Shi, J.-M.; Krolik, J.H. Three-dimensional MHD Simulation of Circumbinary Accretion Disks. II. Net Accretion Rate. Astrophys. J. 2015, 807, 131. [CrossRef]

55. Soker, N. Jet Formation in the Transition from the Asymptotic Giant Branch to Planetary Nebulae. Astrophys. J. 1992, 389, 628. [CrossRef]

56. Zou, Y.; Frank, A.; Chen, Z.; Reichardt, T.; Marco, O.D.; Blackman, E.G.; Nordhaus, J.; Balick, B.; Carroll-Nellenback, J.; Chamandy, L.; et al. Bipolar Planetary Nebulae from Outflow Collimation by Common Envelope Evolution. arXiv 2019, arXiv:1912.01647.

57. Bujarrabal, V.; Castro-Carrizo, A.; Alcolea, J.; Sánchez Contreras, C. Mass, linear momentum and kinetic energy of bipolar flows in protoplanetary nebulae. Astron. Astrophys. 2001, 377, 868. [CrossRef]

58. Blackman, E.G.; Lucchini, S. Using kinematic properties of pre-planetary nebulae to constrain engine paradigms. Mon. Not. R. Astron. Soc. 2014, 440, L16. [CrossRef]

59. Santander-García, M.; Jones, D.; Alcolea, J.; Wesson, R.; Bujarrabal, V. The missing mass conundrum of post-common-envelope planetary nebulae. In Highlights on Spanish Astrophysics X, Proceedings of the XIII Scientific Meeting of the Spanish Astronomical Society, Salamanca, Spain, 16-20 July 2018; Cambridge University Press: Cambridge, UK, 2019; p. 392. 
60. Corradi, R.L.M.; García-Rojas, J.; Jones, D.; Rodríguez-Gil, P. Binarity and the Abundance Discrepancy Problem in Planetary Nebulae. Astrophys. J. 2015, 803, 99. [CrossRef]

61. Brown, A.J.; Jones, D.; Boffin, H.M.J.; Van Winckel, H. On the post-common-envelope central star of the planetary nebula NGC 2346. Mon. Not. R. Astron. Soc. 2019, 482, 4951-4955. [CrossRef]

62. Huggins, P. J. Jets and Tori in Proto-Planetary Nebulae. Astrophys. J. 2007, 663, 342. [CrossRef]

63. Jones, D.; Boffin, H.M.J. Binary stars as the key to understanding planetary nebulae. Nature Astron. $2017,1,0117$. [CrossRef]

64. Sahai, R.; Claussen, M.; Sánchez Contreras, C.; Morris, M.; Sarkar, G. High-Velocity Interstellar Bullets in IRAS 05506+2414: A Very Young Protostar. Astrophys. J. 2008, 680, 483. [CrossRef]

65. Soker, N. Planets, Planetary Nebulae, and Intermediate Luminosity Optical Transients (ILOTs). Galaxies 2018, 6, 58. [CrossRef]

66. Sabach, E.; Soker, N. The class of Jsolated stars and luminous planetary nebulae in old stellar populations. Mon. Not. R. Astron. Soc. 2018, 479, 2249. [CrossRef]

67. Pudritz, R.E.; Hardcastle, M.J.; Gabuzda, D.C. Magnetic Fields in Astrophysical Jets: From Launch to Termination. Space Sci. Rev. 2012, 169, 27. [CrossRef]

68. Balick, B.; Perinotto, M.; Maccioni, A.; Terzian, Y.; Hajian, A. FLIERs and Other Microstructures in Planetary Nebulae. II. Astrophys. J. 1994, 424, 800. [CrossRef]

69. Blackman, E.G.; Frank, A.; Welch, C. Magnetohydrodynamic Stellar and Disk Winds: Application to Planetary Nebulae. Astrophys. J. 2001, 546, 288. [CrossRef]

70. Guidarelli, G.; Nordhaus, J.; Chamandy, L.; Chen, Z.; Blackman, E.G.; Frank, A.; Carroll-Nellenback, J.; Liu, B. Hydrodynamic simulations of disrupted planetary accretion discs inside the core of an AGB star. Mon. Not. R. Astron. Soc. 2019, 490, 1179. [CrossRef]

71. Nordhaus, J.; Blackman, E.G. Low-mass binary-induced outflows from asymptotic giant branch stars. Mon. Not. R. Astron. Soc. 2006, 370, 2004. [CrossRef]

72. Reyes-Ruiz, M.; López, J.A. Accretion Disks in Pre-Planetary Nebulae. Astrophys. J. 1999, 524, 952. [CrossRef]

73. Soker, N. Destruction of Brown Dwarfs and Jet Formation in Planetary Nebulae. Astrophys. J. 1996, 468, 774. [CrossRef]

74. Soker, N. Energy and angular momentum deposition during common envelope evolution. New Astron. 2004, 9, 399. [CrossRef]

75. Hachisu, I.; Kato, M.; Nomoto, K. A Wide Symbiotic Channel to Type IA Supernovae. Astrophys. J. 1999, $522,487$. [CrossRef]

76. Jones, D.; Boffin, H.M.J.; Rodríguez-Gil, P.; Wesson, R.; Corradi, R.L.M.; Miszalski, B.; Mohamed, S. The post-common envelope central stars of the planetary nebulae Henize 2-155 and Henize 2-161. Astron. Astrophys. 2015, 580, A19. [CrossRef]

77. Prialnik, D.; Livio, M. The outcome of accretion on to a fully convective star Expansion or contraction? Mon. Not. R. Astron. Soc. 1985, 216, 37. [CrossRef]

78. Akashi, M.; Kashi, A. Fast ejecta resulted from jet-wind interaction in the Great Eruption of Eta Carinae. 2020, preprint.

79. Grichener, A.; Soker, N. The Common Envelope Jet Supernova (CEJSN) r-process Scenario. Astrophys. J. 2019, 878, 24. [CrossRef]

80. Soker, N.; Grichener, A.; Gilkis, A. Diversity of common envelope jets supernovae and the fast transient AT2018cow. Mon. Not. R. Astron. Soc. 2019, 484, 4972. [CrossRef]

81. Gilkis, A.; Soker, N.; Kashi, A. Common envelope jets supernova (CEJSN) impostors resulting from a neutron star companion. Mon. Not. R. Astron. Soc. 2019, 482, 4233. [CrossRef]

82. Schreier, R.; Hillel, S.; Soker, N. Inclined jets inside a common envelope of a triple stellar system. Mon. Not. R. Astron. Soc. 2019, 490, 4748. [CrossRef]

(C) 2020 by the author. Licensee MDPI, Basel, Switzerland. This article is an open access article distributed under the terms and conditions of the Creative Commons Attribution (CC BY) license (http:/ / creativecommons.org/licenses/by/4.0/). 